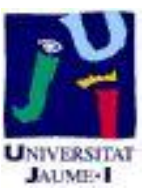

Título artículo / Títol article: Epidemiology of injuries in First Division Spanish football

Autores / Autors

Noya Salces, Javier ; Gómez Carmona, Pedro

M. ; Gracia Marco, Luis ; Moliner Urdiales,

Diego ; Sillero Quintana, Manuel

Revista:

Journal of Sports Sciences Volume 32, Issue 13, 2014

Versión / Versió:

Postprint de l'autor

Cita bibliográfica / Cita

NOYA SALCES, Javier, et al. Epidemiology of injuries bibliogràfica (ISO 690): in First Division Spanish football. Journal of sports sciences, 2014, 32.13: 1263-1270.

url Repositori UJI:

http://hdl.handle.net/10234/127505 


\title{
Epidemiology of injuries in First Division Spanish football
}

\section{JAVIER NOYA SALCES ${ }^{1}$, PEDRO M. GÓMEZ CARMONA ${ }^{2}$, LUIS GRACIA-MARCO ${ }^{3}$, DIEGO MOLINER-URDIALES ${ }^{4} \&$ MANUEL SILLERO-QUINTANA $^{2}$}

\author{
${ }^{1}$ Russian Federation, Moscow, Russia, ${ }^{2}$ Universidad Politécnica de Madrid, Madrid, Spain, ${ }^{3}$ University of Exeter, Exeter, \\ UK and ${ }^{4}$ University faume I, Castellón, Spain
}

(Accepted 18 December 2013)

\begin{abstract}
The aim was to examine the injuries sustained by Spanish football players in the First Division and to compare injury-related variables in the context of both competition and training. The injury data were prospectively collected from 16 teams (427 10 players) using a specific web-based survey during the 2008/2009 season. A total of 1293 injuries were identified (145 were recurring injuries). The overall injury incidence was 5.65 injuries per $1000 \mathrm{~h}$ of exposure. Injuries were much more common during competition than during training (43.53 vs. 3.55 injuries per $1000 \mathrm{~h}$ of exposure, $P<0.05)$. Most of the injuries $(89.6 \%)$ involved the lower extremities, and overuse $(65.7 \%)$ was the main cause. Muscle and tendon injuries were the most common types of injury (53.8\%) among the players. The incidence of training injuries was greater during the pre-season and

15 tended to decrease throughout the season, while the incidence of competition injuries increased throughout the season (all $P<0.05)$. In conclusion, the results of this study suggest the need for injury prevention protocols in the First Division of the Spanish Football League to reduce the number of overuse injuries in the muscles and tendons in the lower extremities. In addition, special attention should be paid during the pre-season and the competitive phase II (the last four months of the season) in order to prevent training and competition injuries, respectively.
\end{abstract}

20 Keywords: professional football, men, injury incidence, injury exposure, football association

\section{Introduction}

The negative impacts that injuries have on the health, performance and finances of football players and clubs are well known. Recent European (Dauty

25 \& Collon, 2011; Ekstrand, Hagglund, \& Walden, 2011b; Hagglund, Walden, \& Ekstrand, 2005) and American (Morgan \& Oberlander, 2001) epidemiological studies of professional male football players reported an overall incidence of approximately 6-8

30 injuries per $1000 \mathrm{~h}$ of exposure. Previous studies have shown that football injuries mainly affected the lower extremities (Wong \& Hong, 2005), particularly the ankle, knee or thigh (Azubuike \& Okojie, 2009; Ekstrand, Hagglund, \& Walden, 2011 a; Junge \& Dvorak, 2004).

In 2011, the First Division of the Spanish Football League was recognised as the strongest national league in the world by the International Federation of Football History and Statistics (IFFHS), and 4 of its 40 teams were ranked among the top 25 in the world. Nonetheless, there are no reference data on injury incidence in the professional Spanish football leagues, and this gap in the data requires urgent attention. Therefore, this prospective study examines the incidence of injuries among male Spanish football players in the First Division during the 2008/ 2009 season and compares injury-related variables in the context of both competition and training.

\section{Materials and methods}

Participants and study period

All of the First Division Spanish football clubs ( $n=20$ ) competing in the $2008 / 2009$ season were contacted and invited to participate in the study. Ultimately, 16 clubs and 427 players $(26.6 \pm 2.6$ players/club) participated; four clubs refused to participate. Medical staff opposition, the time commitment and concerns about confidentiality were the main reasons for not participating. Injuries were recorded from early July 2008 to the end of May 2009. Previous injuries were not taken into consideration but players were included once the injury was healed. Those whose injuries continued to heal after the end of the study period were included, and the duration of the recovery period was discussed with 
65 the respective medical staff. Injuries of those players who were hired by the teams after the study started were not included.

The season included the pre-season (from 6 July 2008 to 23 August 2008) and the competitive season

70 (from 24 August 2008 to 31 May 2009). The competitive season included 2 rounds with 20 teams: competitive phase I (from 24 August 2008 to 18 January 2009) and competitive phase II (from 19 January 2009 to 31 May 2009). All of the matches

75 and training sessions were performed on natural turf surfaces.

\section{Data collection}

The head of the medical staff of every club was initially contacted. Contact information was 80 obtained from the clubs' official websites or the Spanish Football Federation (RFEF). After a brief explanation over the telephone, a letter describing the project was sent to the participants. Written informed consent was obtained from the clubs and

85 players that agreed to participate in the study. The study was performed in accordance with the ethical guidelines of the 1961 Declaration of Helsinki (revision of Edinburgh, 2000). The survey instrument and the research design were approved by the

90 Ethics Committee of the Technical University of Madrid (UPM), Madrid, Spain.

\section{Survey}

A web survey based on the Fédération Internationale de Football Association (FIFA) Consensus Statement (2006) was used to record the type, location and severity of injuries. The medical staff from the clubs reported each injury through the web survey. The database was checked weekly by the researchers, and the medical staff members were contacted when necessary.

\section{Location of injuries}

This study used the following 12 categories of injuries, which have been used in previous studies (Fuller et al., 2006; Hawkins, Hulse, Wilkinson,

105 Hodson, \& Gibson, 2001; Woods, Hawkins, Hulse, \& Hodson, 2002): foot, ankle, lower leg, knee, thigh, hip/groin, upper extremities, shoulder/clavicle, lumbar/sacrum/pelvis, head/face/neck/cervical, abdomen and sternum/rib/dorsal.

\section{Types of injury}

Injuries were classified into seven categories in accordance with the Consensus Statement for football (Fuller et al., 2006): fractures and bone stress, joints (non-bone) and ligaments, muscles and tendons, contusions, lacerations and skin lesions, central/peripheral nervous system and other injuries. In addition, injuries were also classified as traumatic (those with an acute onset) or overuse injuries (those without any known trauma).

\section{Severity of injury}

The severity of each injury was defined according to the number of days elapsed from the date of injury to the date of the player's return to full participation in team training or availability for competition. The injury severity was classified into four categories that have been used in previous studies (Fuller et al., 2006; Woods et al., 2002): minimal ( $\leq 3$ days), mild (4-7 days), moderate (8-28 days) and severe (>28 days). In addition, recurrent injuries were recorded. Recurrent injuries were defined as injuries of the same type and location that occurred after the player recovered and returned to full participation. Recurrent injuries were classified as less severe, equally severe or more severe in comparison to the original injury.

\section{Player exposure and injury incidence}

Three teams were individually followed to obtain mean values to estimate the competition and training exposure of the other teams. Using these values, weekly exposure was estimated on a team basis. Weekly competition exposure, including national (League) and international matches, was calculated by summing the number of competition matches per week for each team and multiplying by $90 \mathrm{~min}$ (the official duration of a match) and 11 players. Players' participation with national teams was not recorded. Weekly training exposure was calculated by multiplying the duration (in minutes) of every training session by the mean number of players (per team). Moreover, the warm-up period before competition matches (25 $\min \times 11$ players) and friendly matches (90 $\min \times 11$ players), which were mainly played within the own team, were also considered as training exposure as in previous studies (Walden, Hagglund, \& Ekstrand, 2005b). Cool down periods after competition matches were not usual among Spanish teams and were not taken into account for exposure calculation. Finally, the results were divided by 60 to obtain competition and training exposure in hours. The injury incidence is expressed as the number of injuries per $1000 \mathrm{~h}$ of training or competition.

\section{Statistical analysis}

Descriptive statistics are presented as frequencies, proportions (\%), incidence rates (per $1000 \mathrm{~h}$ of 
165 exposure) and 95\% confidence intervals (non-overlapping confidence intervals indicate a significant difference between competition and training) (Kirkwood \& Sterne, 2003). Lost days due to an injury are presented as mean and standard deviation

170 (minimum and maximum values are indicated when standard deviation is greater than mean). All of the analyses were performed using the PASW statistical package, version 18.0 (SPSS Inc., Chicago, IL), with $P<0.05$ considered statistically significant.

\section{Results}

The mean age of the players was $26.8 \pm 4.1$ years, their height was $181 \pm 6 \mathrm{~cm}$ and their weight was $75.8 \pm 5.7 \mathrm{~kg}$. A total of 1293 injuries were reported by the 427 players ( 524 competition injuries and 769

180 training injuries). The top 3 injuries were 1) thigh muscle injuries; $31.4 \%, 11.5$ days of time loss (min: 1, max: 190); 2) ankle sprain; $12.5 \%, 9.7$ days of time loss (min: 1, max: 94); and 3) hip muscle injuries; $10.9 \%, 8.6$ days of time loss (min: 1, max: 82).

The total exposure was $228,743 \mathrm{~h}(12,038 \mathrm{~h}$ of competition exposure and $216,705 \mathrm{~h}$ of training exposure). Overall injury incidence was 5.65 (CI 5.35-5.96) injuries per $1000 \mathrm{~h}$ of exposure. The

190 overall incidence of injuries was greater in competition (43.53; CI 39.96-47.42) than in training (3.55; CI 3.31-3.81). Incidences were also greater in competition independently of location, type and/or nature. In addition, the incidence of recurrent injuries

195 was also greater in competition (4.7; CI 3.65-6.14) than in training $(0.4 ; \mathrm{CI} 0.33-0.5)(P<0.05)$.

\section{Injury location}

Of all injuries, $89.6 \%$ involved the lower extremities (injury incidence $=5.1$ ). The highest incidence of 200 injuries affected the thigh, followed by the ankle, hip/ groin, knee and lower leg (Table I).

\section{Injury type}

Muscle and tendon injuries had the highest incidence rate (3.04), followed by injuries of the joints and ligaments (1.38) and contusions (0.8). More than $50 \%$ of the injuries were related to muscles and tendons (both competition- and training-related injuries) (Table II).

\section{Injury nature}

210 Two out of three injuries were due to overuse (59.5\% during competition and $70.0 \%$ during training), and one out of three injuries was due to trauma (40.5\% during competition and $30.0 \%$ during

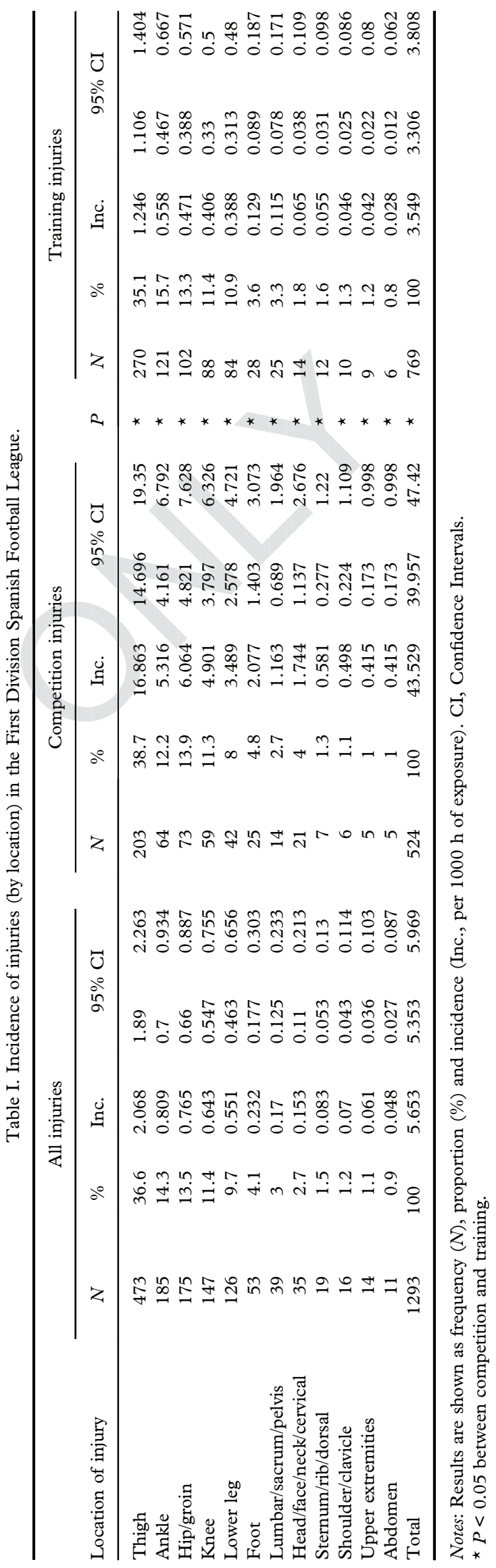


training). The incidence rates of overuse injuries and traumatic injuries were greater during competition than during training (25.9 and 17.6 vs. 2.5 and 1.1 injuries per $1000 \mathrm{~h}$; all $P<0.05)$. Both overuse $(65.7 \%)$ and traumatic injuries $(34.3 \%)$ were related to an average time loss per injury of 11.4 days (min: 1, max: 215 and min: 1, max: 247, respectively) (Table III).

\section{Injury severity and recurrence}

Injuries of all degrees of severity occurred more commonly in competition than in training (all $P<0.05)$. Most frequent injuries involved minimal $(35.7 \%, 2.2 \pm 0.7$ days of time loss), mild (26.8\%, $5.1 \pm 1.1$ days of time loss) and moderate severity $(29.2 \%, 14.5 \pm 5.6$ days of time loss) (table IV).

Figure 1 shows the incidence rates of recurrent injuries according to their severity. The incidence of recurrent injuries was greater during competition $(0.8-2.2)$ than during training $(0.1-0.3)$ (all $P<0.05)$. In addition, equally severe and more severe recurrent injuries occurred during competition with greater frequency $(2.2$ and 1.6 , respectively) than less severe injuries (0.8).

\section{Injury incidence per month}

Figure 2 shows the incidence of training injuries per month throughout the season. The incidence of injuries in the pre-season (July and August) was greater than the incidence during the rest of the season $(P<0.05)$. In addition, more injuries occurred during competitive phase I than during competitive phase II $(P<0.05)$. The incidence of training injuries tended to decrease throughout the season.

Figure 3 shows the incidence of injuries that occurred during competition each month throughout the season. The incidence of injuries in the competitive phase II was greater than the incidence in the competitive phase I $(P<0.05)$. Despite a progressive increase from September, the incidence of injuries dropped in December and especially in April, peaking in March and May.

\section{Discussion}

To our knowledge, this is the first study examining the incidence and the characteristics of injuries among the players in a large number of Spanish teams competing in the First Division. The main findings of this study indicate that (1) competition causes more injuries than training; (2) the top 3 injuries were thigh muscle injuries, ankle sprain and hip muscle injuries; (3) competition is associated with a greater severity of injuries than training; and

\section{5}


Table III. Incidence of injuries (by nature) in the First Division Spanish Football League.

\begin{tabular}{|c|c|c|c|c|c|c|c|c|c|c|c|c|c|c|c|c|}
\hline \multirow{3}{*}{$\begin{array}{l}\text { Nature of injury } \\
\text { Overuse }\end{array}$} & \multicolumn{5}{|c|}{ All injuries } & \multicolumn{5}{|c|}{ Competition injuries } & \multirow[b]{2}{*}{$\mathrm{P}$} & \multicolumn{5}{|c|}{ Training injuries } \\
\hline & $N$ & $\%$ & Inc. & $95 \%$ & CI & $N$ & $\%$ & Inc. & $95 \%$ & $\mathrm{CI}$ & & $N$ & $\%$ & Inc. & $95 \%$ & CI \\
\hline & 850 & 65.7 & 3.716 & 3.474 & 3.974 & 312 & 59.5 & 25.918 & 23.196 & 28.959 & $\star$ & 538 & 70.0 & 2.483 & 2.281 & 2.702 \\
\hline Traumatic & 443 & 34.3 & 1.937 & 1.764 & 2.126 & 212 & 40.5 & 17.611 & 15.393 & 20.149 & $\star$ & 231 & 30.0 & 1.066 & 0.937 & 1.213 \\
\hline
\end{tabular}

Notes: Results are shown as frequency $(N)$, proportion (\%) and incidence (Inc., per $1000 \mathrm{~h}$ of exposure). CI, Confidence Intervals. $\star P<0.05$ between competition and training.

Table IV. Incidence of injuries (by severity) in the First Division Spanish Football League.

\begin{tabular}{|c|c|c|c|c|c|c|c|c|c|c|c|c|c|c|c|c|}
\hline \multirow{3}{*}{$\begin{array}{l}\text { Severity } \\
\text { Minimal }\end{array}$} & \multicolumn{5}{|c|}{ All injuries } & \multicolumn{5}{|c|}{ Competition injuries } & \multirow[b]{2}{*}{$\mathrm{P}$} & \multicolumn{5}{|c|}{ Training injuries } \\
\hline & \multirow{2}{*}{$\frac{N}{462}$} & \multirow{2}{*}{$\begin{array}{c}\% \\
35.7\end{array}$} & \multirow{2}{*}{$\begin{array}{c}\text { Inc. } \\
2.020\end{array}$} & \multicolumn{2}{|c|}{$95 \% \mathrm{CI}$} & \multirow{2}{*}{$\frac{N}{155}$} & \multirow{2}{*}{$\begin{array}{c}\% \\
29.6\end{array}$} & \multirow{2}{*}{$\begin{array}{c}\text { Inc. } \\
12.876\end{array}$} & \multicolumn{2}{|c|}{$95 \% \mathrm{CI}$} & & \multirow{2}{*}{$\frac{N}{307}$} & \multirow{2}{*}{$\frac{\%}{39.9}$} & \multirow{2}{*}{$\frac{\text { Inc. }}{1.417}$} & \multicolumn{2}{|c|}{$95 \% \mathrm{CI}$} \\
\hline & & & & 1.844 & 2.213 & & & & 11.000 & 15.071 & $\star$ & & & & 1.267 & 1.584 \\
\hline Mild & 346 & 26.8 & 1.513 & 1.361 & 1.681 & 153 & 29.2 & 12.710 & 10.847 & 14.892 & $\star$ & 193 & 25.1 & 0.891 & 0.773 & 1.026 \\
\hline Moderate & 377 & 29.2 & 1.648 & 1.490 & 1.823 & 169 & 32.2 & 14.039 & 12.074 & 16.323 & $\star$ & 208 & 27.1 & 0.960 & 0.838 & 1.100 \\
\hline Severe & 108 & 8.3 & 0.472 & 0.391 & 0.570 & 47 & 9.0 & 3.904 & 2.933 & 5.196 & $\star$ & 61 & 7.9 & 0.281 & 0.219 & 0.362 \\
\hline
\end{tabular}

Notes: Results are shown as frequency $(N)$, proportion (\%) and incidence (Inc., per $1000 \mathrm{~h}$ of exposure). CI, Confidence Intervals.

$\star P<0.05$ between competition and training.

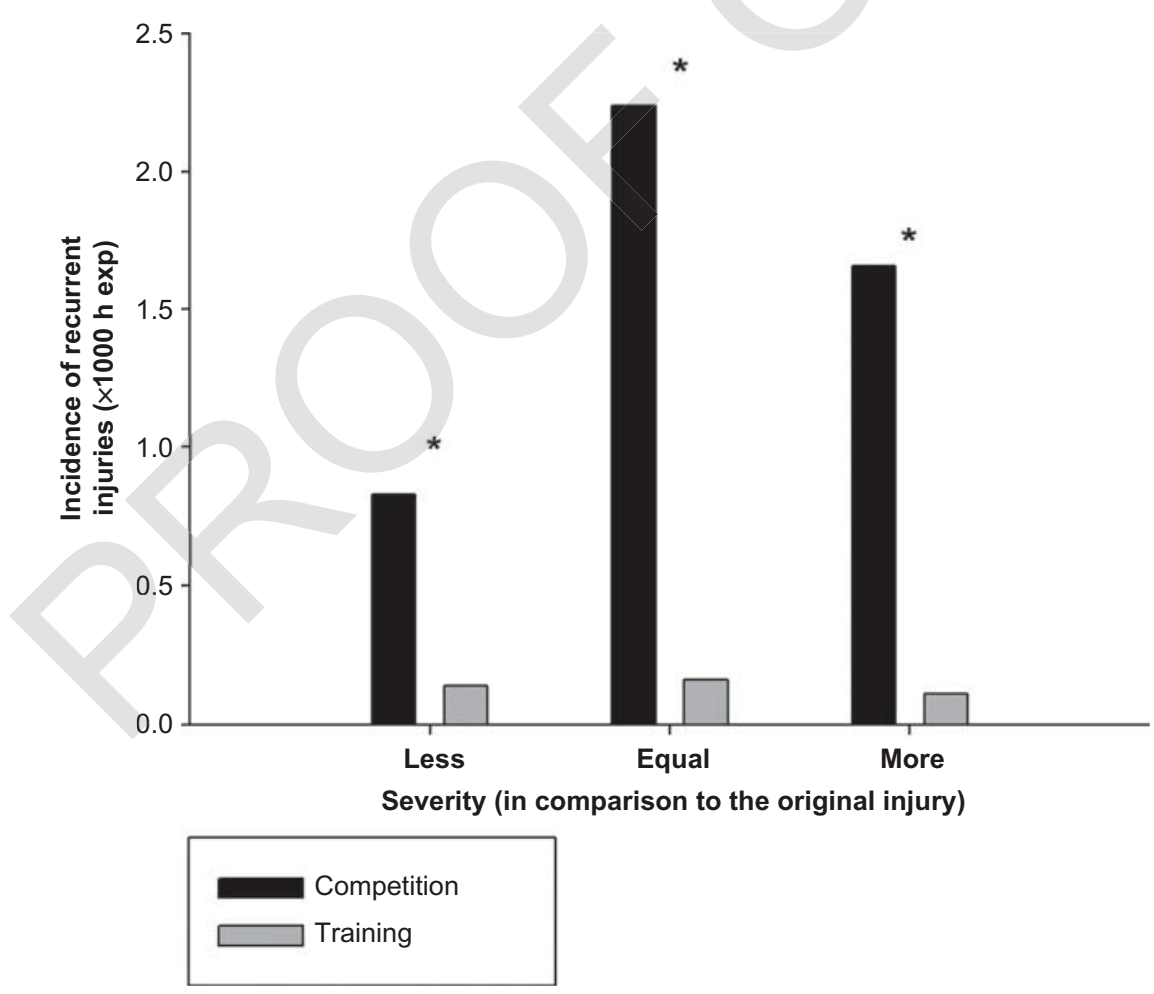

Figure 1. Incidence of recurrent injuries according to severity in the First Division Spanish Football League. ${ }^{\star} P<0.05$ between competition and training.

(4) the incidence of injuries during training decreases throughout the season, whereas the incidence of injuries during competition increases.
First, our results showed that injury incidence was 12.3 times greater in competition than in training (43.53 vs. 3.55), as previous studies have shown. 


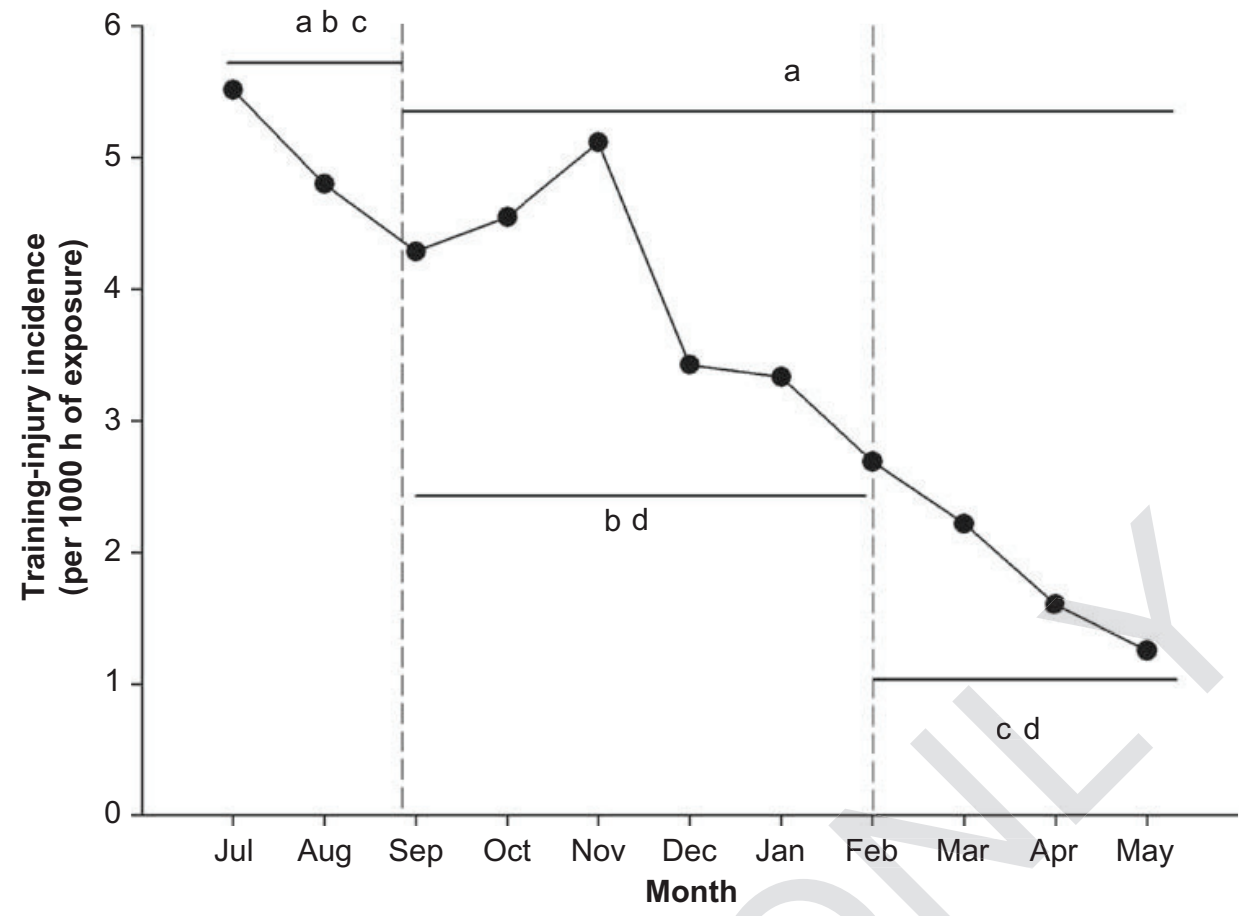

Figure 2. Monthly incidence of training injuries according to the phases of the season for the First Division Spanish Football League. ${ }^{\text {abcd }}$ Common superscripts indicate a significant difference.

${ }^{\mathrm{a}} P<0.05$ between pre-season and rest of the season.

${ }^{\mathrm{b}} P<0.05$ between pre-season and competitive phase I.

${ }^{\mathrm{c}} P<0.05$ between pre-season and competitive phase II.

${ }^{\mathrm{d}} P<0.05$ between competitive periods I and II.

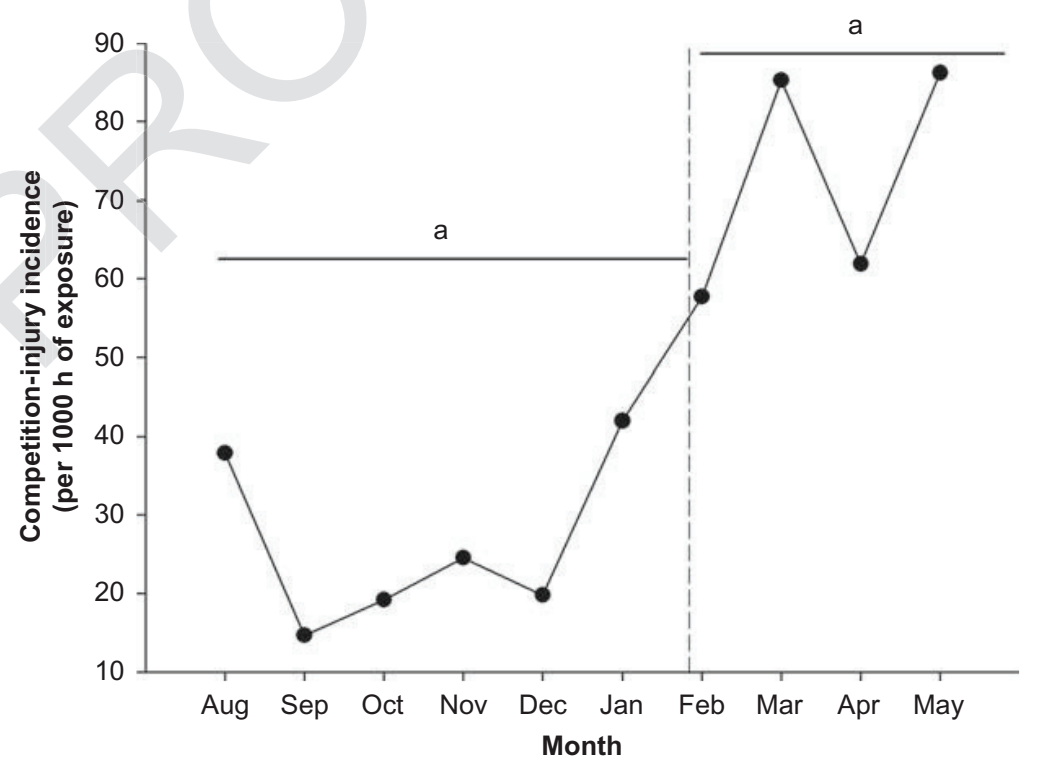

Figure 3. Monthly incidence of competition injuries according to the phases of the season for the First Division Spanish Football League. ${ }^{\mathrm{a}} \mathrm{P}<0.05$ between competitive phase I and II. 
Morgan and Oberlander (2001) showed that competition was associated with 12.2 times more injuries than training (35.3 vs. 2.9) in the American Major Soccer (Football) League. Recently, Ekstrand et al. (2011b) studied 23 of the 50 best European teams (2001-2008) and showed that competition was associated with 6.7 times more injuries than training (27.5 vs. 4.1). Hagglund et al. (2005) showed that the incidence of injuries was greater in competition than in training in the top Danish (28.2 vs. 11.8$)$ and Swedish (26.2 vs. 6.0) male football divisions. These important differences between competition and training may occur because the intensity is greater during competition than during training, which creates a higher risk of injury. In addition, other factors such as the calculation of exposure time or even different levels of competitiveness during matches may explain these differences between studies.

Second, according to location our results showed the highest incidence of injuries to the lower extremities, particularly the thigh, followed by the ankle, hip/ groin and knee. These results based on Spanish players align with the results based on European (Ekstrand et al., 2011b; Hagglund et al., 2005; Hawkins et al., 2001; Walden et al., 2005b) and American male professional football players (Morgan \& Oberlander, 2001). According to type, we found results similar to previous studies where the highest incidences of injury were related to muscles and tendons, joints and ligaments, and contusions (Ekstrand et al., 2011b; Hagglund et al., 2005; Hawkins et al., 2001; Walden et al., 2005b). According to nature, we

300 showed that overuse injuries were more prevalent $(66 \%)$ and had twice the incidence rate (3.7 vs. 1.9$)$ of traumatic injuries, with incidence rates being greater during competition than during training.

Third, our results showed that the most reported 305 injuries $(63 \%)$ were of minimal or mild ( $\leq 7$ days) severity, while severe injuries ( $>28$ days) only represented $8 \%$ of the total number of injuries. The current scientific literature on modern football shows a slightly greater severity of injuries than our study 310 found (Drawer \& Fuller, 2002; Ekstrand et al., 2011b; Hawkins et al., 2001; Morgan \& Oberlander, 2001; Walden et al., 2005b). Ekstrand et al. (2011b) found that $50 \%$ of injuries were at least of moderate severity $(37 \%$ in our study with 8-28 days out of competition). Similarly, Hawkins et al. (2001) studied football players from the four professional English leagues and showed that $68 \%$ of injuries involved more than 8 days out of competition. The results from the UEFA Champions League and the Major Football League showed that $15 \%$ of injuries were severe (Morgan \& Oberlander, 2001; Walden et al., 2005b). It is likely that differences in training protocols and styles of play among countries could explain the slightly lower severity of injuries in the Spanish league. In addition, $11 \%$ of the injuries were classified as recurrent injuries, as previous European studies have found (Ekstrand, Hagglund, \& Walden, 2010; Ekstrand et al., 2011a; Walden, Hagglund, \& Ekstrand, 2005a, 2005b). These data suggest the need to improve the criteria required for players to return to play, as well as the rehabilitation processes, to reduce the risk of recurrent injuries (Fuller \& Walker, 2006). In this regard, designing functional tests in order to check the progress of the players during the rehabilitation process is a research topic that needs more emphasis.

Finally, our data showed significant differences in injury incidence depending on the phase of the season, as previous studies have found (Dauty \& Collon, 2011; Ekstrand et al., 2011b; Hawkins et al., 2001; Morgan \& Oberlander, 2001; Walden et al., 2005b; Woods et al., 2002). In the present study, the highest incidence of training injuries was observed during the pre-season, most likely because of inadequate recovery between seasons and/or inappropriate demands during the pre-season. For some players trying to secure a place in the first squad might be associated with an increased injury risk, as their intensity during training could be higher. The incidence of injuries during competition peaked during competitive phase II. This increase might be explained by deconditioning and/or by an increase in the intensity of play because of the importance of the results, which are crucial during this phase of the season (Morgan \& Oberlander, 2001).

\section{Study limitations and strengths}

The main limitation of the study is the estimation of the exposure time. Both competition exposure and training exposure were estimated on a weekly basis (to avoid recall bias, a daily estimate would have been preferable) based on the mean number of players per team (not on the actual number of players present at a particular club event). Moreover, time exposure with national teams was not obtained for the present study. Therefore, we consider that exposure time could be underestimated. In relation to injures, those with duration of zero days, and some types such as abrasions, were not recorded. In addition, the players' histories of previous injuries, and injuries with national teams, were not taken into account. Therefore, we may have missed some additional data on injuries. As a consequence of this, the real injury incidence could slightly differ from the one showed in our results. The study design, which mainly followed the recommendations of the FIFA Consensus Statement (Fuller et al., 2006), and the large homogeneous sample of elite male football players (427 players from 16 teams in the First Division Spanish Football League) are the main strengths of our study. 


\section{Conclusions}

Injury incidence in the First Division Spanish Football League is similar to other European Leagues. Competition causes more injuries and is associated with injuries of greater severity than train-

385 ing. In addition, it is important to note the differences in injury incidence between competition and training in each phase of the season. Injuries caused by overuse and thigh-related injuries were the most common injuries among the players. Special atten-

390 tion should be paid during the pre-season and the competitive phase II in order to prevent training and competition injuries, respectively.

Further studies analysing changes in injury incidence between different seasons and using more accurate exposure time recording are needed to confirm our data and to establish specific prevention protocols for the First Division Spanish Football.

\section{References}

Azubuike, S. O., \& Okojie, O. H. (2009). An epidemiological study of football (soccer) injuries in Benin City, Nigeria. British fournal of Sports Medicine, 43, 382-386.

Dauty, M., \& Collon, S. (2011). Incidence of injuries in French professional soccer players. International fournal of Sports Medicine, 32, 965-969.

405 Drawer, S., \& Fuller, C. W. (2002). Evaluating the level of injury in English professional football using a risk based assessment process. British Fournal of Sports Medicine, 36, 446-451.

Ekstrand, J., Hagglund, M., \& Walden, M. (2010). Injury incidence and injury patterns in professional football: The UEFA injury study. British fournal of Sports Medicine, 45, 553-558.

Ekstrand, J., Hagglund, M., \& Walden, M. (2011a). Epidemiology of muscle injuries in professional football (soccer). American fournal of Sports Medicine, 39, 1226-1232.
Ekstrand, J., Hagglund, M., \& Walden, M. (2011b). Injury incidence and injury patterns in professional football: The UEFA injury study (Research support, non-U.S. Gov't). British Fournal of Sports Medicine, 45, 553-558.

Fuller, C. W., \& Walker, J. (2006). Quantifying the functional rehabilitation of injured football players. British fournal of Sports Medicine, 40, 151-157.

Fuller, C. W., Ekstrand, J., Junge, A., Andersen, T. E., Bahr, R., Dvorak, J., ... Meeuwisse, W. H. (2006). Consensus statement on injury definitions and data collection procedures in studies of football (soccer) injuries. British Fournal of Sports Medicine, 40, 193-201.

Hagglund, M., Walden, M., \& Ekstrand, J. (2005). Injury incidence and distribution in elite football - a prospective study of the Danish and the Swedish top divisions. Scandinavian fournal of Medicine E Science in Sports, 15, 21-28.

Hawkins, R. D., Hulse, M. A., Wilkinson, C., Hodson, A., \& Gibson, M. (2001). The association football medical research programme: An audit of injuries in professional football. British Fournal of Sports Medicine, 35, 43-47.

Junge, A., \& Dvorak, J. (2004). Soccer injuries: A review on incidence and prevention. Sports Medicine, 34, 929-938.

Kirkwood, B. R., \& Sterne, J. A. C. (2003). Essential medical statistics (2nd ed.). Oxford: Blackwell Publishing.

Morgan, B. E., \& Oberlander, M. A. (2001). An examination of injuries in major league soccer: The inaugural season. The American fournal of Sports Medicine, 29, 426-430.

Walden, M., Hagglund, M., \& Ekstrand, J. (2005a). Injuries in Swedish elite football - a prospective study on injury definitions, risk for injury and injury pattern during 2001. Scandinavian fournal of Medicine E Science, 15, 118-125.

Walden, M., Hagglund, M., \& Ekstrand, J. (2005b). UEFA Champions League study: A prospective study of injuries in professional football during the 2001-2002 season. British fournal of Sports Medicine, 39, 542-546.

Wong, P., \& Hong, Y. (2005). Soccer injury in the lower extremities. British Fournal of Sports Medicine, 39, 473-482.

Woods, C., Hawkins, R., Hulse, M., \& Hodson, A. (2002). The Football Association Medical Research Programme: An audit of injuries in professional football-analysis of preseason injuries. British Fournal of Sports Medicine, 36, 436-441.

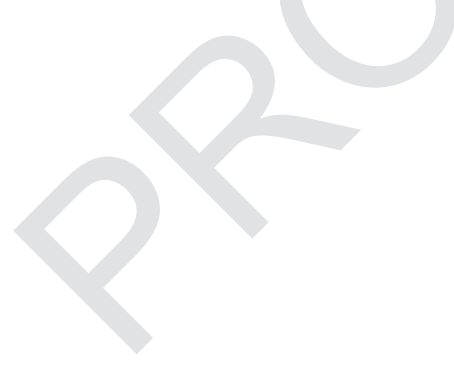

